\title{
Neuroanesthesia: A Problem-Based Learning Approach
}

\author{
David E. Traul, Irene P. Osborn (Editors). Oxford University Press 2019, Published in \\ the United States of America, 249 pages. ISBN 978-0-19-085003-6
}

\author{
Michael Dinsmore, BSc, PhD, MD, FRCPC (D)
}

Received: 31 January 2019/Accepted: 31 January 2019/Published online: 12 February 2019

(C) Canadian Anesthesiologists' Society 2019

Neuroanesthesia: A Problem-Based Learning Approach is a succinct (249 pages), case-based textbook that provides a comprehensive review of the subspecialty neuroanesthesia. The 29 case-based chapters are organized into eight major sections, including commonly performed procedures (oncological, vascular, spine, pediatric, functional, and other), perioperative complications, and important neuroanesthesia concepts.

Each chapter is well structured and designed to follow the oral board examination format. It begins with a familiar neuroanethesia case description that progresses smoothly from basic learning points to more complex complications the reader may encounter. The case-based questions provide an effective guide for reviewing key didactic concepts such as neuroanatomy, physiology, and pharmacology while maintaining clinical relevance. The discussion section that follows each case is a complete review that offers a comprehensive, up-to-date examination of the chapter's subject matter. In most instances, the discussion progresses logically through each case by first examining the preoperative considerations and patient optimization. It then proceeds with patient monitoring and positioning, intraoperative concerns, and complications, finally addressing emergence from anesthesia and postoperative disposition. Each chapter ends with ten review questions and answers that highlight key concepts and help consolidate the

\footnotetext{
M. Dinsmore, BSc, PhD, MD, FRCPC ( $₫)$

Faculty of Medicine, University of Toronto, Toronto, ON,

Canada

e-mail: michael.dinsmore@uhn.ca
}

information. The questions are also available online in a full practice examination format for flexibility of their use.

The various sections are organized in a thoughtful manner, with the first chapter of each section beginning with a common procedure (e.g., craniotomy for a supratentorial tumour). The authors effectively incorporate many of the fundamental neuroanesthesia topics (premedication, ventilation, cerebral blood flow, cerebral metabolic rate, intracranial pressure, cerebral perfusion pressure, use of osmotic diuretics, choice of anesthetic agents, differential diagnosis for delayed emergence), providing the basic building blocks to draw upon while progressing through the textbook and tackling more complex problems. Although the various chapters focus on commonly performed procedures and their associated problems, the textbook achieves a more complete picture by including neurosurgical considerations for both pediatric and pregnant populations. In addition, it addresses more specialized procedures such as awake craniotomy, extracranial-intracranial bypass, and deep brain stimulation.

Throughout the textbook, the authors incorporate valuable insights and provide evidence-based information on a number of topics, such as proper maintenance fluids, the use of steroids and hypothermia for traumatic brain injury, and the use of video laryngoscopy for unstable cervical spine surgery. The information is complete, up-to-date, and referenced appropriately. The authors' use of detailed color illustrations and radiographic imaging are visually appealing and provide additional information for better clinical comprehension.

In summary, Neuroanesthesia: A Problem-Based Learning Approach is a highly useful textbook. It effectively uses problem-based learning to engage the reader. Well written, easy to read, and well organized, it provides valuable information that is properly referenced 
and coalesces the information needed to improve clinical understanding and practice. Each case progresses from the basic to the more complex topics, making it a valuable resource for both inexperienced neuroanesthesiologists and as a reminder of already known subjects for more seasoned practitioners who are seeking a refresher text. I recommend this book as both a useful reference manual and an excellent educational resource.
Conflicts of interest None declared.

Editorial responsibility This submission was handled by $\mathrm{Dr}$ Hilary P. Grocott, Editor-in-Chief, Canadian Journal of Anesthesia.

Publisher's Note Springer Nature remains neutral with regard to jurisdictional claims in published maps and institutional affiliations. 